\title{
Fístula traqueoesofágica associada a paracoccidioidomicose
}

\section{Tracheoesophageal fistula associated with paracoccidioidomicosis}

Antonio Carlos Nogueira ${ }^{a}$, Daniel Soares de Sousa Dantas ${ }^{\mathrm{b}}$, Francisco Garcia Soriano ${ }^{\mathrm{a}}$, Simone Peres Pillic, João Epalanga Júnior Vidro ${ }^{d}$, Edmar Tafner ${ }^{c}$, Lincoln Tavares de Andrade ${ }^{c}$, Luis Masuo Marutac ${ }^{c}$, Hélio Minamoto ${ }^{e}$, José Pinhata Otoch ${ }^{f}$, João Augusto dos Santos Martines ${ }^{g}$

Nogueira AC, Dantas DSS, Soriano FG, et al. Fístula traqueoesofágica associada a paracoccidioidomicose. Autopsy Case Rep [Internet]. 2011;1(3):31-8. http://dx.doi.org/10.4322/acr.2011.006

\section{RESUMO}

A paracoccidioidomicose é uma micose sistêmica causada pelo Paracoccidioides brasiliensis, que é encontrado em regiões da América do Sul e Central, com casos relatados do norte do México ao sul da Argentina. As infecções causadas pelo $P$. brasiliensis frequentemente mimetizam outras doenças. A paracoccidioidomicose pulmonar crônica é frequentemente confundida com neoplasia ou tuberculose. Os autores apresentam o caso de um homem de 57 anos internado com quadro de tosse crônica, fraqueza, disfagia, mal estar e síndrome consumptiva. Fora submetido a tratamento para tuberculose com rifampicina, pirazinamida e isoniazida há 1 ano sem melhora dos sintomas. Durante a investigação clínica foi diagnosticada paracoccidioidomicose pulmonar com fistula traqueo-esofágica. A infecção foi tratada com anfotericina $B$ deoxicolato e posteriormente com sulfametoxazol e trimetoprim devido a desenvolvimento de insuficiência renal aguda. A fístula foi tratada endoscopicamente com implante de prótese traqueal para proteger o brônquio esquerdo, seguido de clipagem do óstio esofágico da fístula.

Unitermos: Paracoccidioidomicose; Fístula traqueo-esofágica; Próteses e implantes.

\section{ABSTRACT}

Paracoccidioidomycosis is a systemic fungal disease caused by Paracoccidioides brasiliensis, agent geographically distributed to certain areas of Central and South America. The infection by $P$. brasiliensis has been reported from north Mexico to south Argentina. Paracoccidioidomycosis presents similar clinical findings of many other diseases whatever in acute or

\footnotetext{
a Divisão de Clínica Médica do Hospital Universitário - Universidade de São Paulo, São Paulo/SP - Brasil.

${ }^{\mathrm{b}}$ Instituto de Infectologia Emílio Ribas, São Paulo/SP - Brasil.

c Serviço de Endoscopia do Hospital Universitário - Universidade de São Paulo, São Paulo/SP - Brasil.

${ }^{\text {d }}$ Medico estagiário do Hospital das Clínicas da Faculdade de Medicina - Universidade de São Paulo, São Paulo/SP - Brasil.

e Hospital das Clínicas da Faculdade de Medicina - Universidade de São Paulo, São Paulo/SP - Brasil.

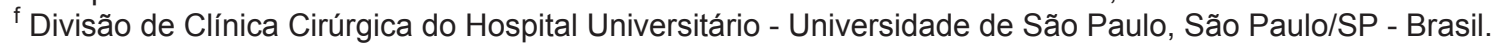

g Serviço de Imagenologia do Hospital Universitário - Universidade de São Paulo, São Paulo/SP - Brasil.
}

Copyright () 2011 Autopsy and Case Reports - Este artigo de Acesso Aberto é distribuído pelos termos do Creative Commons Attribution NonCommercial License (http://creativecommons.org/licenses/by/3.0/) que permite livre uso não-comercial, distribuição e reprodução em qualquer meio, desde que os artigos sejam devidamente citados. 
chronic scenarios. Chronic pulmonary paracoccidioidomycosis is frequently misdiagnosed as malignancy or tuberculosis. The authors present a case of a 57 year-old man admitted to the hospital due to a chronic consumptive syndrome. Heunderwent anti-tuberculous treatment with rifampin, isoniazid and pyrazinamide 1 year ago without resolution of the simptoms. During the clinical investigation, pulmonary paracoccidioidomycosis with tracheoesophageal fistula was diagnosed. The systemic infection was treated with deoxicolate $B$ amphotericin followed by sulfametoxazole and trimetoprin due to acute renal function impairment. The fistula was endoscopically treated; inittialy with the protection of left main bronchus with a tracheal prosthesis followed by the esophageal fistula's ostium clipping.

Keywords: Paracoccidioidomycosis; Tracheoesophageal fistula; Prostheses and implants.

\section{RELATO DO CASO}

Homem, 57 anos, natural da Bahia, procedente de São Paulo - SP, aposentado, trabalhou na agricultura e como servente de pedreiro, procurou o pronto-socorro com queixa de tosse produtiva de aspecto purulento há 3 semanas. Associado ao quadro referia perda ponderal não quantificada nos últimos meses, anorexia, disfagia, astenia e constipação intestinal há 1 semana. Negava febre, hemoptise, vômitos, melena, hematêmese, dor abdominal, icterícia, colúria ou acolia fecal, lesões tegumentares ou alterações neurológicas. Relatava como único antecedente mórbido a história de tuberculose pulmonar tratada adequadamente há 1 ano. Tabagista e etilista há 30 anos tendo consumido, neste período, 1 litro de aguardente diariamente. Ao exame físico da admissão apresentava-se em regular estado geral, moderadamente descorado e desidratado, afebril, acianótico, anictérico, eupnéico, caquético. O exame pulmonar revelou tórax sem alterações à inspeção, murmúrio vesicular presente, simétrico com roncos e estertores difusos e bilaterais. A ausculta cardíaca mostrava ritmo cardíaco regular em dois tempos, bulhas rítmicas e normofonéticas, sem sopros. Os pulsos periféricos eram amplos, simétricos e sincrônicos. $O$ abdome estava distendido, flácido, porém com leve dor à palpação profunda difusamente sem sinal de Blumberg, não foram palpadas visceromegalias, nem observados sinais de insuficiência hepática. Os membros inferiores não apresentavam edema nem tampouco sinais de trombose venosa profunda. As hipóteses diagnósticas levantadas foram neoplasia e tuberculose, sendo solicitados exames complementares.
Os exames laboratoriais mostraram: hemoglobina $=11,6 \mathrm{~g} \%$, com índices hematimétricos compatíveis comanemia normocítica, normocrômica, leucócitos $=6500 \cdot \mathrm{mm}^{-3}$ (bastonetes $=6 \%$, segmentados $=83 \%$, eosinófilos $=1 \%$, linfócitos $=8 \%$, monócitos $=2 \%$ ) e Sódio $=132 \mathrm{mEq} \cdot \mathrm{L}^{-1}$, sem outras alterações. A pesquisa de bacilo álcool-ácido resistente no escarro foi negativa em 3 amostras. A sorologia para HIV foi negativa. A radiografia de tórax (Figura 1) revelou opacidade retículo-nodular difusa bilateral com discreto predomínio nos campos pulmonares superiores e peri hilar bilateral. Solicitada tomografia computadorizada de tórax que evidenciou lesão compatível com fístula traqueoesofágica acima do arco aórtico (Figuras 2 e 3), linfadenomegalia paratraqueal com gânglios de $1,5 \mathrm{~cm}$ de diâmetro sugerindo necrose/liquefação central (Figura 4) e a presença de fístulas adeno brônquicas bilaterais (Figura 3 ). O parênquima pulmonar exibia múltiplas imagens cavitárias com paredes espessadas predominando os lobos superiores e múltiplos nódulos disseminados além de bronquiectasias. A distribuição do comprometimento pulmonar é peri-hilar bilateral com aspecto de "asa de borboleta" (Figura 5).

Após o diagnóstico de fístula traqueoesofágica foram solicitadas endoscopia digestiva alta (EDA) e broncoscopia para elucidação etiológica da mesma. A EDA mostrou lesão ulcerada e infiltrativa de aproximadamente $3 \mathrm{~cm}$ em esôfago proximal, a cerca de $20 \mathrm{~cm}$ da arcada dentária superior. Observou-se orifício fistular no fundo da ulceração (Figuras 6 e 7). A análise da micro-vascularização por cromoscopia eletrônica e magnificação de imagem foi sugestiva de provável lesão neoplásica. Devido à presença de fístula, não foi possível efetuar cromoscopia com lugol. $A$ 


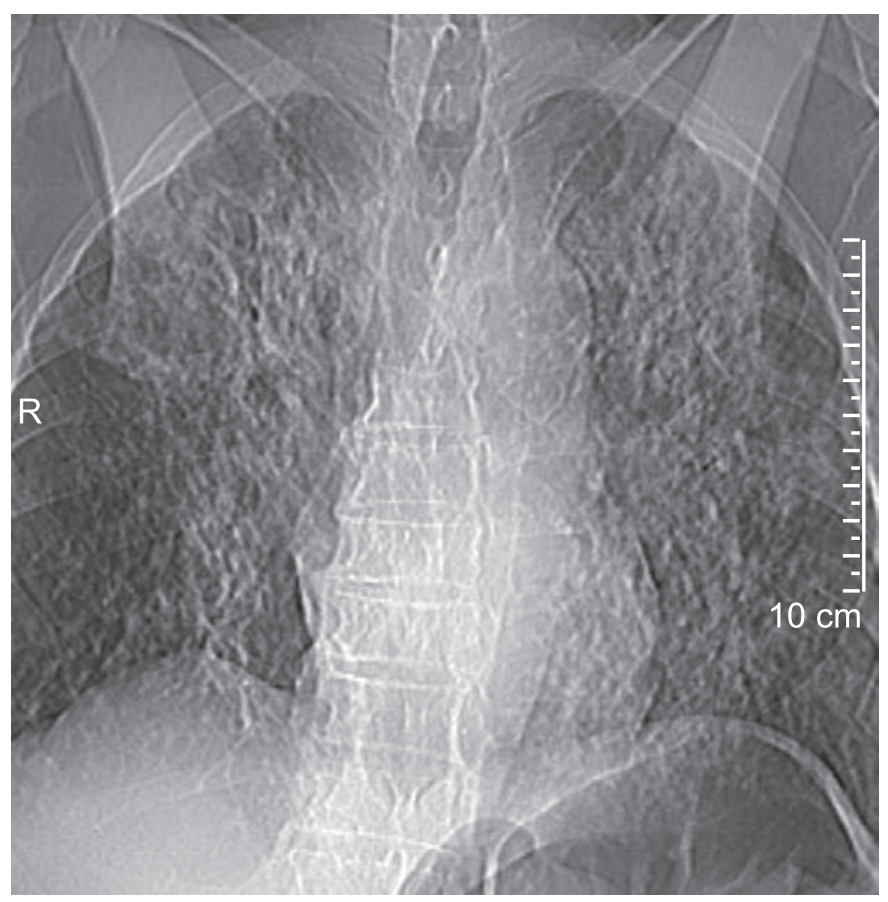

Figura 1 - Radiografia de tórax em PA - opacidade retículo-nodular difuso bilateral com discreto predomínio nos campos pulmonares superiores.

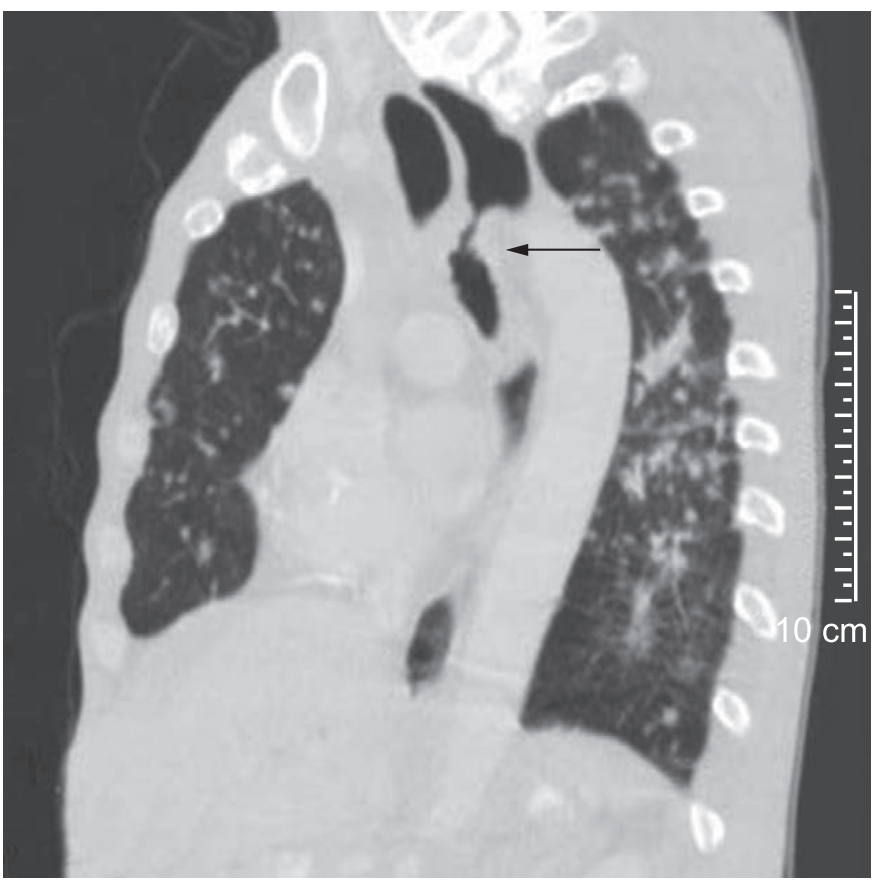

Figura 2 - Tomografia de tórax - corte sagital evidenciando fístula traqueo-esofágica acima do arco aórtico.
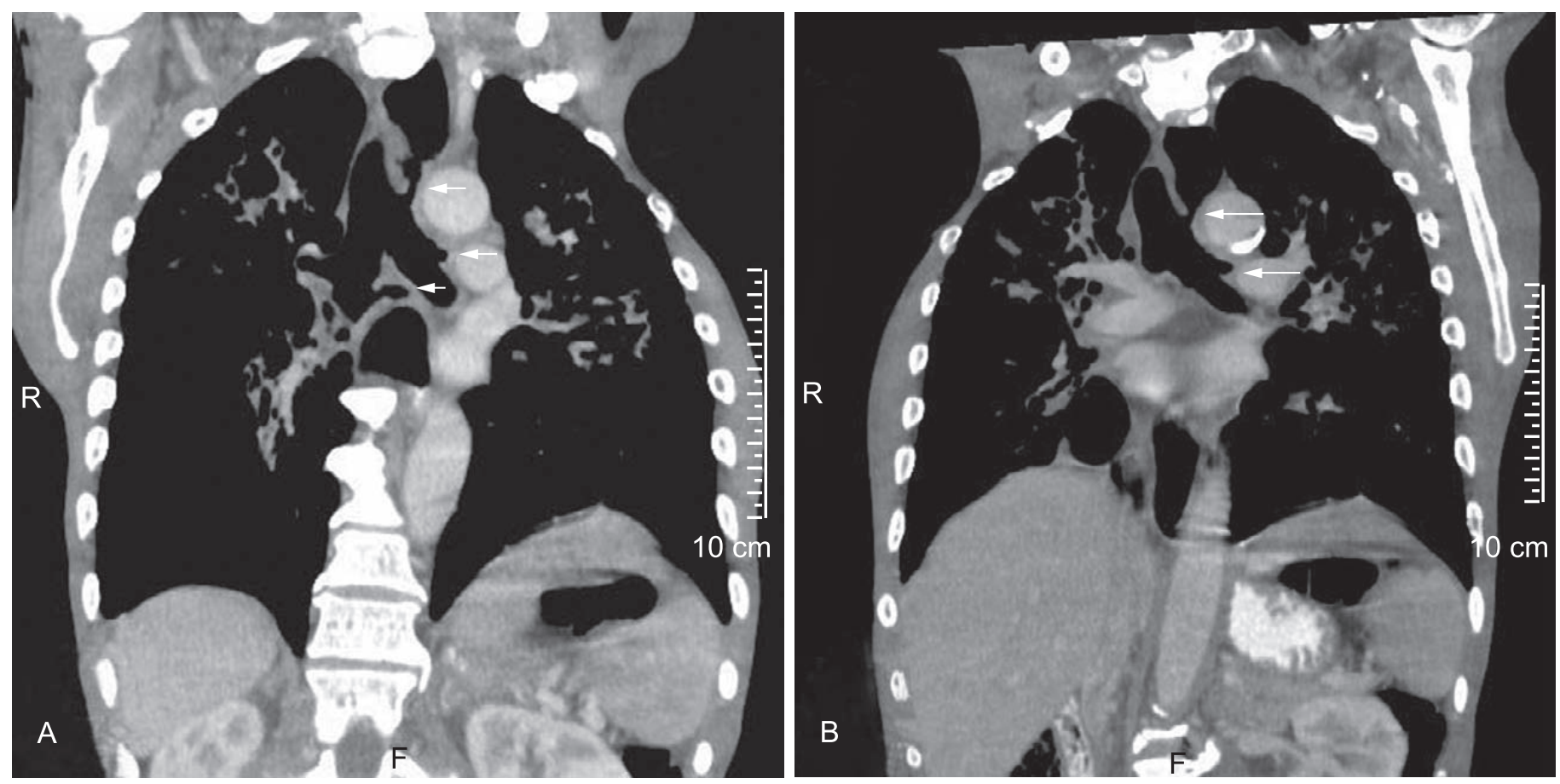

Figura 3 - Tomografia de tórax A e B cortes coronais, janela de mediastino evidenciando fístula traqueoesofágica (seta superior), fístula para linfonodos (setas inferiores).

mucosa do restante do esôfago se mostrava com aspecto normal em terço médio e distal. A transição esofagogástrica, o estômago e o duodeno não apresentavam alterações endoscópicas, exceto por focos de enantema e depósito de hematina indicando sangramento recente em mucosa de antro.
A broncoscopia revelou processo inflamatório/infiltrativo extenso, acometendo a região distal da traquéia, carina principal e brônquio principal esquerdo. Foi observado aspecto infiltrativo circular da mucosa na emergência do brônquio principal esquerdo com orifício fistular de aproximadamente 
$6 \mathrm{~mm}$ além de processo inflamatório intenso nos segmentos distais. Brônquios do pulmão direito com processo inflamatório moderado (Figura 8).

O estudo histopatológico das biópsias do esôfago revelou processo inflamatório crônico granulomatoso com presença de fungos compatíveis com Paracoccidioides brasiliensis (Figura 9).

Devido a extensão da lesão e a gravidade do caso, com insuficiência respiratória (após o procedimento endoscópico) optou-se inicialmente

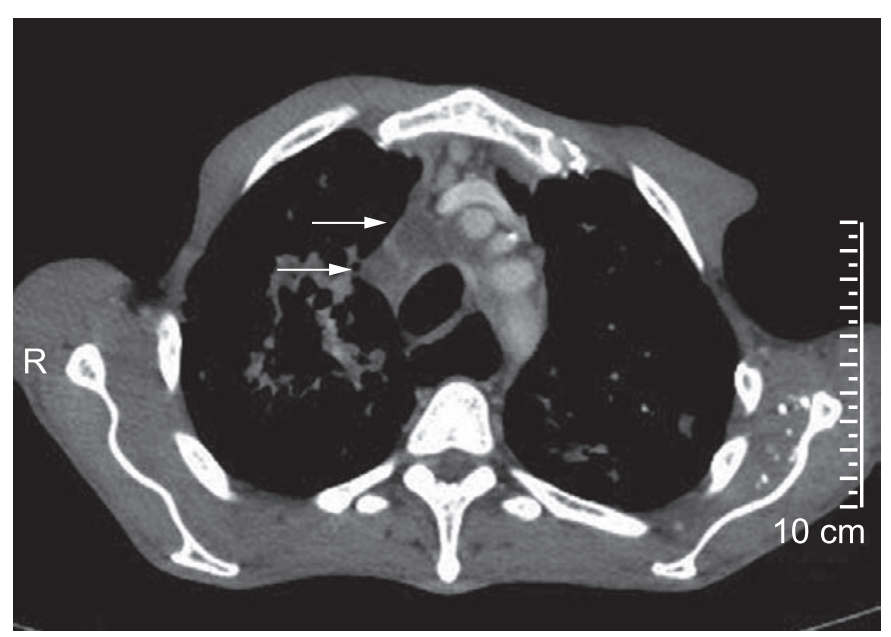

Figura 4 - Tomografia de tórax - corte axial, janela para mediastino revelando a presença de linfonodos paratraqueais liquefeitos (setas).

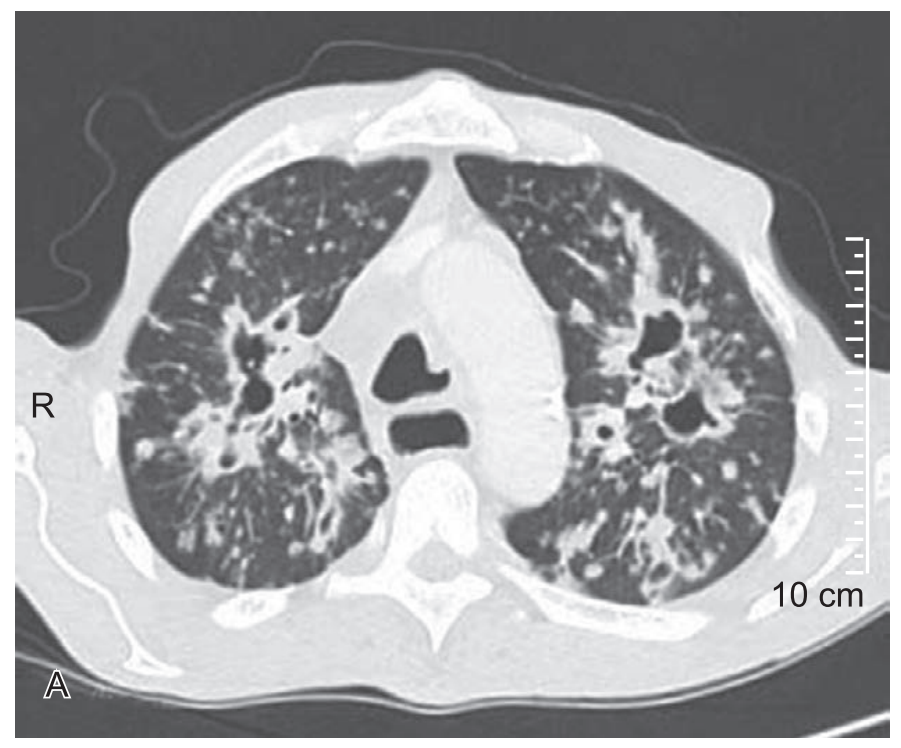

pelo tratamento com anfotericina B deoxicolato, porém em decorrência da progressiva piora da função renal, optou-se pela substituição por sulfametoxazol-trimetoprim, que foi mantido após alta hospitalar. A fístula traqueo-esofágica foi corrigida com o implante de prótese de traquéia para manter a permeabilidade do brônquio fonte esquerdo, evitando o seu colabamento. Com a redução do calibre do óstio e da friabilidade tecidual, decorrente da terapêutica específica, foi possível a clipagem endoscópica por via esofágica (Figuras 10, 11 e 12).

\section{DISCUSSÃO}

A paracoccidioidomicose (PCM) é a infecção fúngica sistêmica de maior prevalência na América Latina. $^{1,2}$ É uma doença infecciosa não-contagiosa adquirida por via inalatória. ${ }^{1}$

O agente etiológico é o fungo dimórfico Paracoccidioides brasiliensis, cujo nicho ecológico ainda não está totalmente estabelecido, predomina em áreas rurais de vários países latino-americanos, sendo o Brasil a principal área endêmica. As regiões sul, sudeste e centro-oeste do Brasil são as áreas de maior prevalência da doença. ${ }^{2-4}$

A forma aguda ou subaguda também chamada de forma juvenil é responsável por 3 a $5 \%$ dos casos da doença, predominando em crianças

Figura 5 - Tomografia de tórax: A - corte axial, B corte coronal revelando múltiplas imagens cavitárias com paredes espessadas predominando os lobos superiores e múltiplos nódulos disseminados, bronquiectasias. A distribuição do comprometimento pulmonar é peri-hilar bilateral com aspecto de "asa de borboleta". 


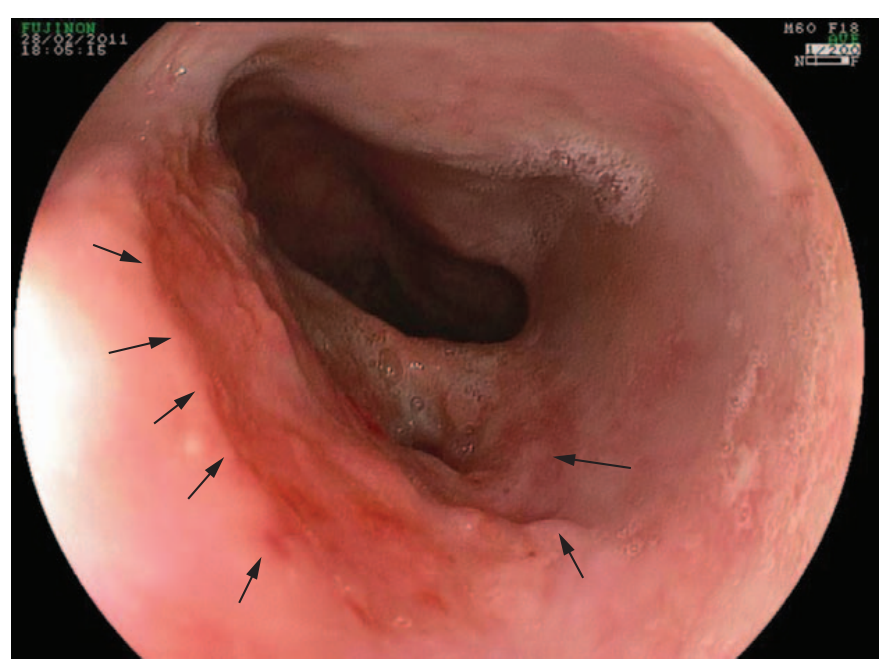

Figura 6 - Imagem de endoscopia em esôfago proximal revela área de enantema e ulceração em esôfago de aproximadamente $3 \mathrm{~cm}$ com bordas irregulares, friável e aspecto infiltrativo. Observase orifício próximo à borda inferior direita da lesão (orifício fistular).

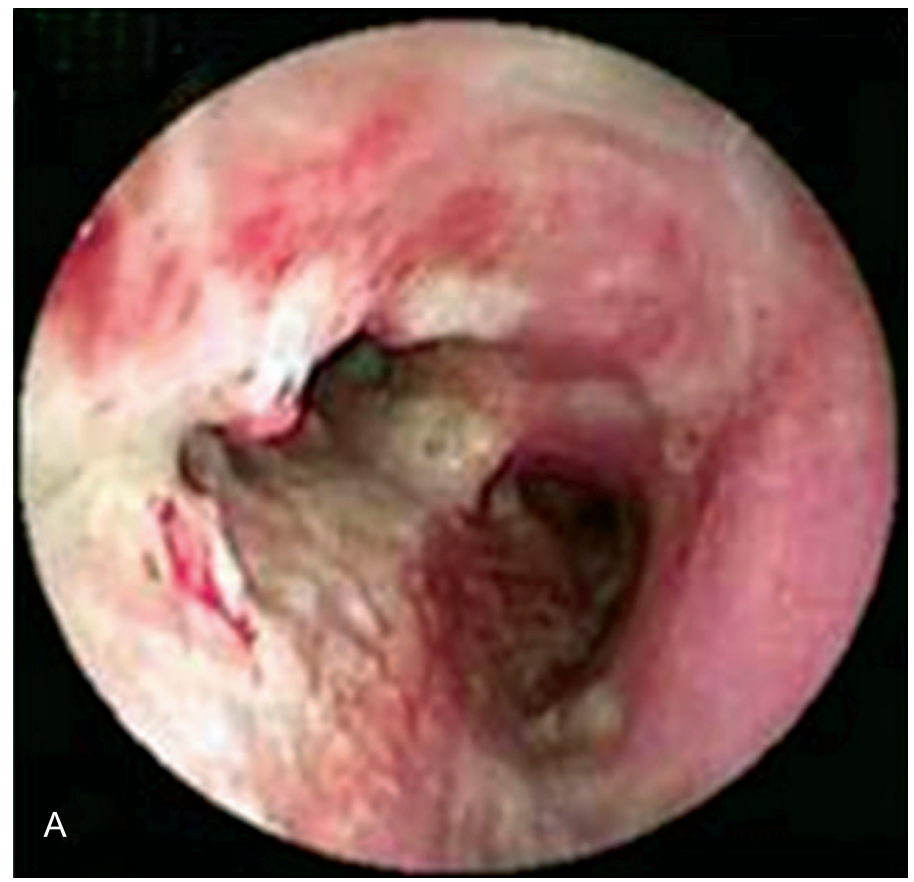

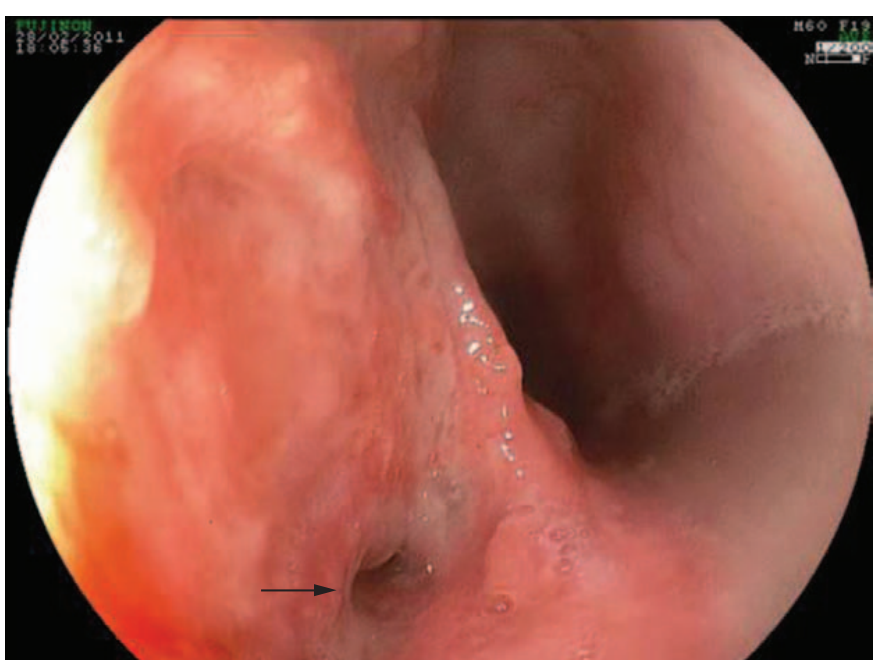

Figura 7 - Imagem endoscópica demonstrando área de ulceração e irregularidade mucosa do esôfago com orifício fistular nítido na borda inferior (seta).

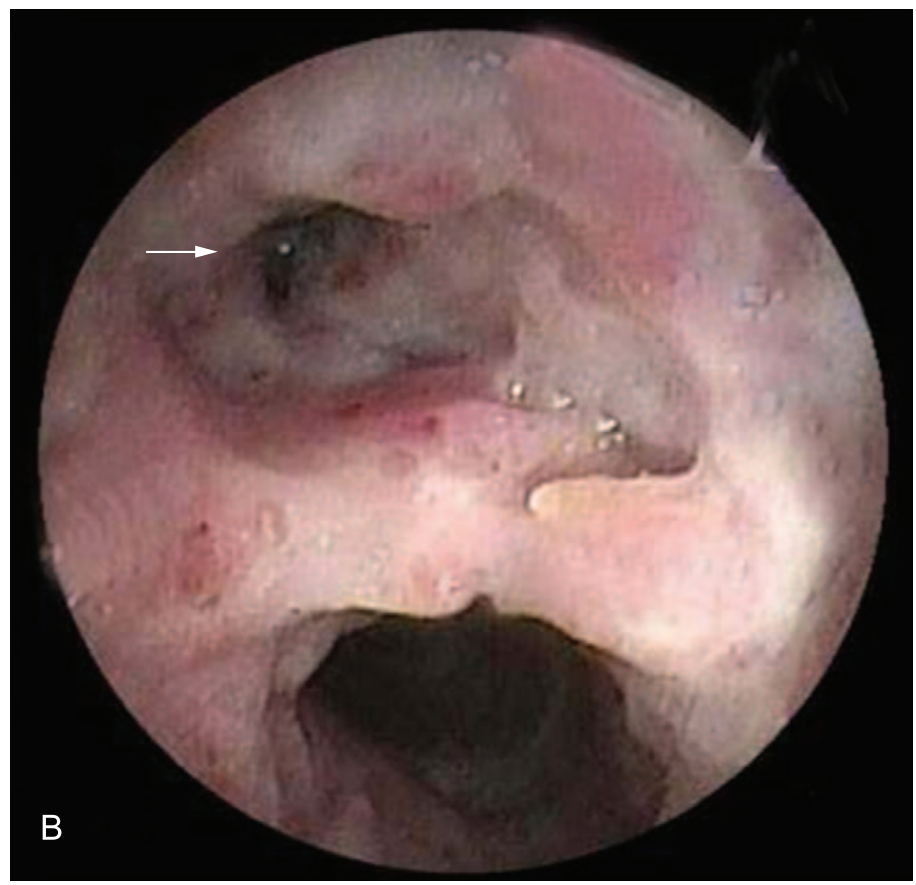

Figura 8 - Imagem broncoscópica demonstra: A - área de irregularidade da mucosa em região distal da traquéia que se prolonga a jusante. Nota-se depósito de muco e aspecto infiltrativo da mucosa envolvendo traquéia distal, carina e brônquios principais direito e esquerdo; B - Imagem do brônquio principal esquerdo com acometimento difuso e com visualização de orifício fistular na parede superior - comunicação com gânglio peri brônquico (seta branca).

e adolescentes. Caracteriza-se por evolução mais rápida, com aparecimento em torno de 4 a 12 semanas de linfadenomegalia, manifestações digestivas, hepatoesplenomegalia, envolvimento ósteo-articular e lesões cutâneas. ${ }^{2,3}$
A forma crônica responde por mais de $90 \%$ dos pacientes, e apresenta-se principalmente em homens entre os 30 e 60 anos na proporção de 10:1 a 15:1 entre homens e mulheres. O estrogênio parece fornecer efeito protetor, de 

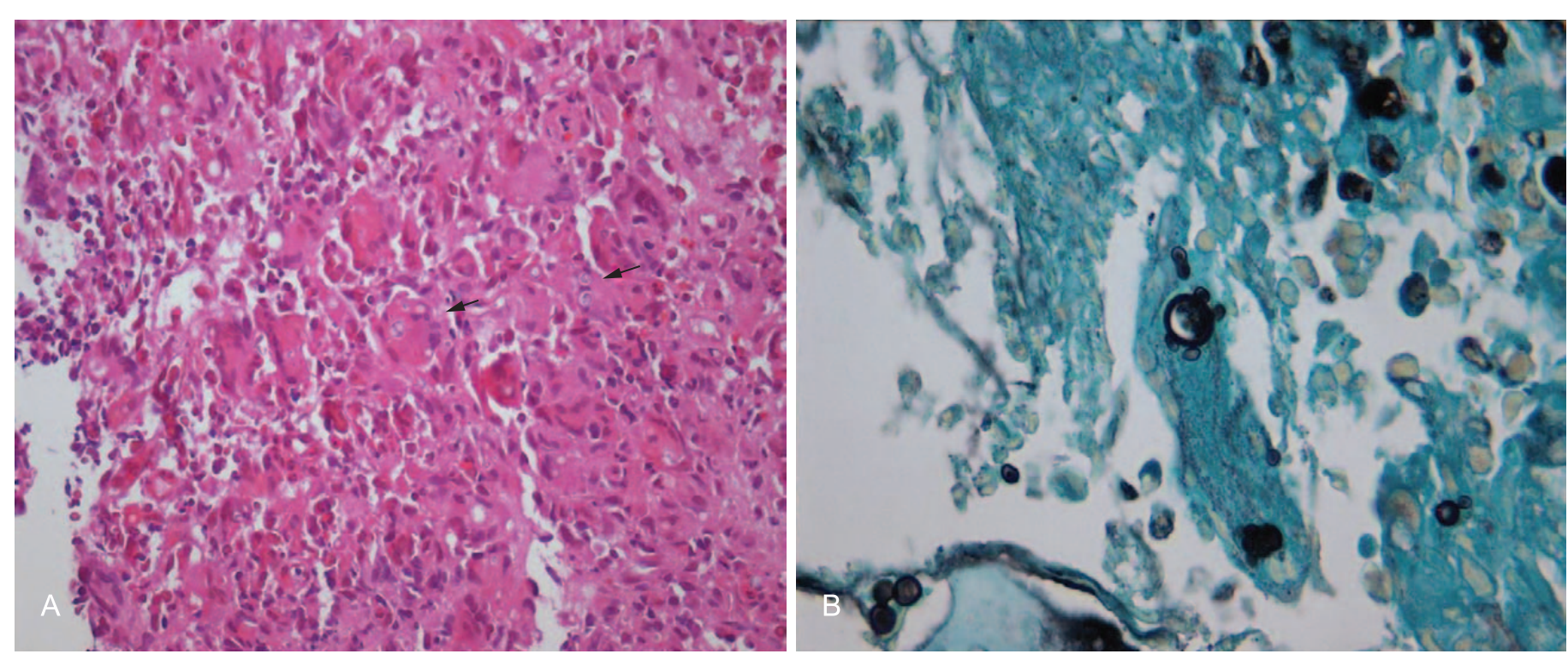

Figura 9 - Fotomicrografias: A - Processo inflamatório com numerosas células gigantes e estruturas fúngicas refringentes (setas) (H\&E, 400X); B - Fotomicrografia de estrutura fúngica com múltiplas gemulações (Grocott, 1000X).
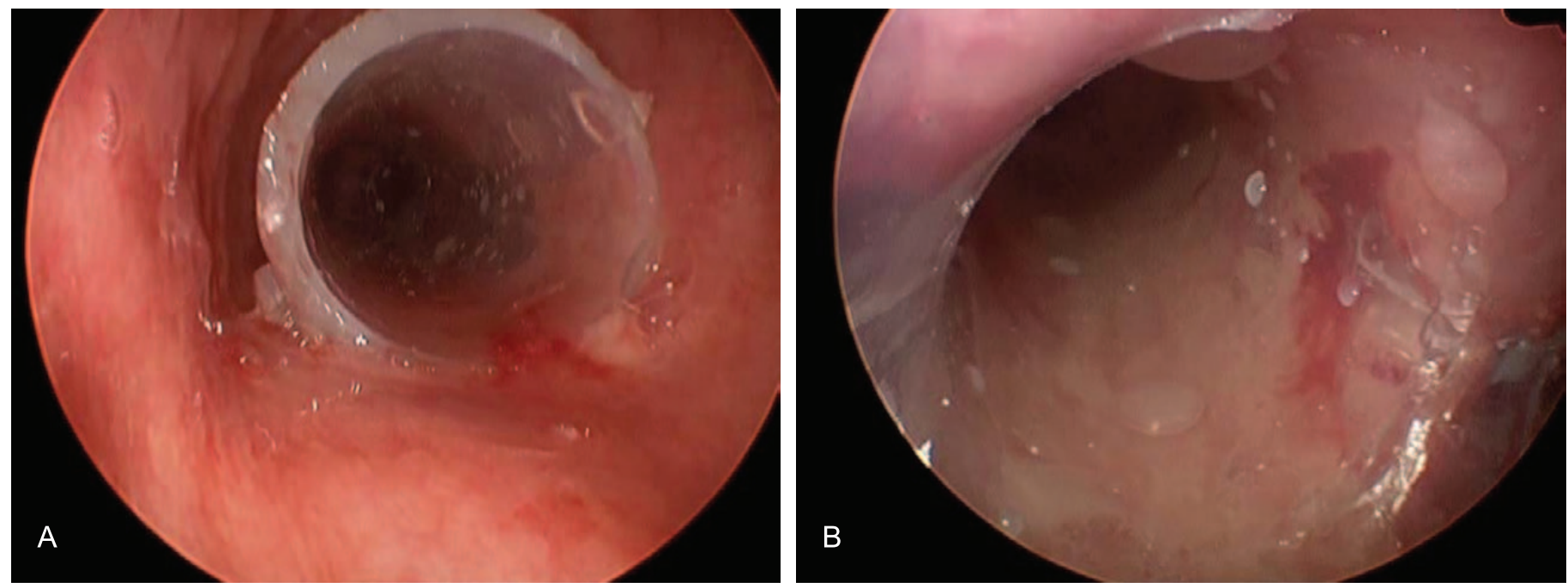

Figura 10 - Visão endoscópica: A - prótese posicionada na traquéia; B - fibroscópio posicionado dentro da prótese entrando no brônquio principal esquerdo que se encontra protegido do colabamento pela prótese.

modo que a prevalência da doença é igual até os 13 anos de idade e, a seguir, torna-se cada vez maior nos homens, até atingir a proporção acima mencionada. ${ }^{1,4-6}$ A doença progride lentamente, de forma silenciosa, podendo levar anos até que seja diagnosticada. ${ }^{1}$ As manifestações pulmonares estão presentes em $90 \%$ dos pacientes, sendo os pulmões o único órgão afetado em até $25 \%{ }^{1}$ dos casos. Entre os diagnósticos diferenciais da PCM crônica pulmonar destacam-se a tuberculose (que pode estar associada em $5-15 \%$ dos casos $)^{2,4}$, sarcoidose, neoplasias, histoplasmose, pneumocistose e pneumoconioses. ${ }^{1,4}$
A forma crônica da PCM representa a reativação endógena anos após o contato inicial com o fungo. A causa da reativação é desconhecida, mas pode estar relacionada a estados de imunossupressão como desnutrição, tabagismo e etilismo crônico ${ }^{4}$, além de outras patologias que alteram o sistema imune como neoplasia, AIDS, drogas imunossupressoras e transplante de órgãos. ${ }^{1}$

Geralmente, a PCM envolve mais de um órgão simultaneamente (apresentação multifocal), sendo pulmões, mucosas e pele os sítios mais acometidos pela infecção. ${ }^{2,3}$ 
O acometimento do tubo digestivo pela paracoccidioidomicose foi descrito desde as primeiras autópsias relatadas em $1945^{7}$. Martinez et al. realizaram estudo para avaliação das lesões do esôfago, estômago e duodeno através de EDA, em uma população de 30 pacientes com doença em atividade independente do seu quadro clínico ou da presença de queixas gastrointestinais. Cerca de $40 \%$ apresentaram lesões diversas e inespecíficas do esôfago, estômago e duodeno. O exame histopatológico do tecido biopsiado não mostrou lesões específicas no esôfago e no estômago confirmando a raridade do comprometimento destes órgãos. Porém o $P$. brasiliensis foi observado em três casos de lesões duodenais. ${ }^{7}$

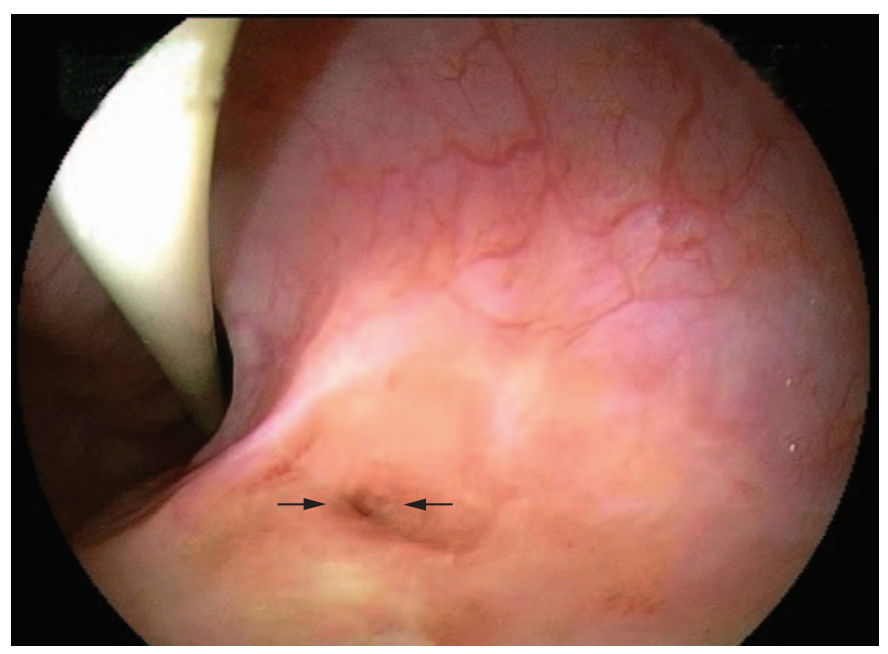

Figura 11 - Visão endoscópica do orifício fistular do esôfago após tratamento específico, mostrando redução do processo inflamatório infiltrativo e redução do diâmetro orificial.
Em outro estudo, Sales et al. ${ }^{8}$ realizaram broncoscopia em 20 pacientes portadores de paracoccidioidomicose, sendoque $75 \%$ dospacientes apresentavam traqueobronquite, provavelmente relacionadas ao tabagismo e 3 apresentavam lesões sugestivas de comprometimento pela PCM. Lesões destrutivas de laringe que fazem diagnóstico diferencial com neoplasias foram descritas em um estudo. $^{9}$

O $P$. brasiliensis é sensível à maioria dos antifúngicos sistêmicos disponíveis, incluindo os derivados sulfamídicos. Os índices de cura variam de 69 a $100 \% .^{10}$ A terapêutica da PCM deve incluir medidas de suporte às complicações clínicas associadas ao envolvimento de diferentes órgãos pela micose além da terapêutica antifúngica específica.

Neste relato, o paciente foi inicialmente tratado com anfotericina $\mathrm{B}$ deoxicolato devido à gravidade do quadro clínico, e substituído posteriormente por sulfametoxazol-trimetoprim devido à piora progressiva da função renal. Outra opção terapêutica, para casos leves e moderados em gravidade, é o itraconazol. ${ }^{1,2,6} \mathrm{Em}$ geral os tratamentos são longos e sua duração depende da resposta clínica, radiológica e imunológica. ${ }^{1}$ Ressaltamos que a cura dos pacientes portadores de PCM nunca será totalmente completa devido à impossibilidade de erradicação de $P$. brasiliensis ${ }^{1,2}$ Os pacientes apresentam o risco potencial de reativação tardia, motivo pelo qual se emprega o termo "cura aparente ou cura clínica". Após a interrupção do tratamento e observados os critérios de cura, os pacientes devem ser acompanhados no ambulatório, pelo menos uma vez ao ano. ${ }^{1}$
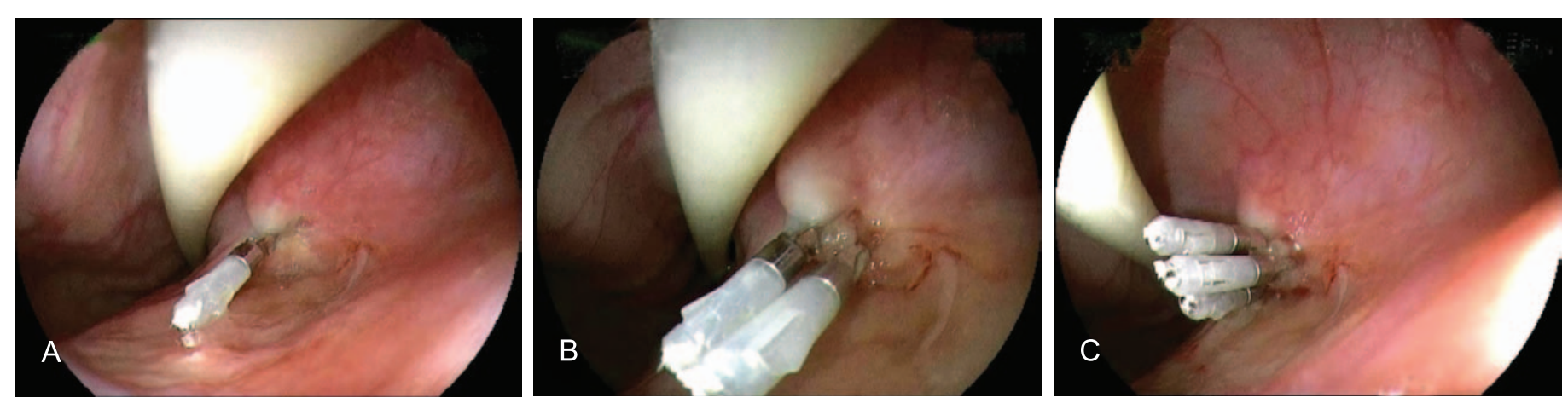

Figura 12 - Visão endoscópica do esôfago mostrando a sequência (A, B e C) de clipagem do orifício fistular - tratamento final da fístula. 


\section{REFERÊNCIAS}

1. Barbosa W, Daher RD. Blastomicose Sul-americana (Paracoccidioidomicose). In: Vernoesi R, Focaccia R, Dietze R. Doenças infecciosas e parasitárias. 8. ed. Rio de Janeiro; Guanabara Koogan; 1991. p. 634-46. Portuguese.

2. Yasuda MAS, Telles Filho FQ, Mendes RP, Colombo AL, Moretti ML, Grupo de Consultores do Consenso em Paracoccidioidomicose. Consenso em paracoccidioidomicose. Rev Soc Bras Med Trop. 2006;39(3):297-310. Portuguese. PMid:16906260.

3. Kauffman CA. Paracoccidioidomycosis. In: Goldman L, Ausiello D, editors. Cecil medicine. 23rd ed. Philadelphia: Saunders Elsevier; 2008. p. 2346-7.

4. Restrepo A, Tobón AM. Paracoccidioides brasiliensis. In: Mandel GL, Bennett JE, Dolin R, editors. Mandell, Douglas, and Bennett's principles and practice of infectious diseases.7th ed. Philadelphia: Churchill Livingstone Elsevier; 2010. v.2, p. 3357-63.

5. Bennett JE. Miscellaneous mycoses and algal Infections. In: Braunwald E, Hauser SL, Fauci AS, Longo DL, Kasper
DL, editors. Harrison's principles of internal medicine. $15^{\text {th }}$ ed. New York: McGraw Hill; 2001. p.1180-1.

6. Nucci $\mathrm{M}$, Colombo AL, Queiroz-Telles F. Paracoccidioidomycosis. Curr Fungal Infect Rep. 2009;3(1):15. http://dx.doi.org/10.1007/s12281-009-0003-0

7. Martinez R, Modena JLP, Barbieri Neto J, Fiorillo AM. Avaliação endoscópica do compromentimento do esôfago,estomago e duodeno na paracoccidiodomicose humana. Arq Gastroenterol. 1986;23(1):21-5. Portuguese.

8. Sales, LHM, Manco JC, Martinez R. Achados de broncoscopia em pacientes com formas polares da paracccidiodomicose. Rev Par Med. 2001;15(4):7-11. Portuguese.

9. Sant'anna GD, Mauri M, Arrarte JL, Camargo HJR. Laryngeal manifestations of paracoccidiodomycosis (South American Blastomycosis). Arch Otolaryngol Head Neck Surg. 1999;125(12):1375-8.

10. Travassos LR, Taborda CP, Colombo AL. Treatment options for paracoccidioidomycosis and new strategies investigated. Expert Rev Anti Infect Ther. 2008;6(2):251-62. PMid:18380607. http://dx.doi.org/10.1586/14787210.6.2.251

Conflito de interesse: Não há.

Submetido em: 16 de Maio de 2011

Aceito em: 18 de Agosto de 2011

Correspondência: Daniel Soares de Sousa Dantas R. Cássio Martins Vilaça, 430 - São Paulo/SP - Brasil CEP: 01249-000 - Tel.: +55 (11) 8258-9794

E-mail: danielssdantas@hotmail.com 